Programa de Pós-Graduação em Engenharia de Produção - PPGEP

Laboratório de Qualidade de Vida - LaQVida

Universidade Tecnológica Federal do Paraná - UTFPR

Ponta Grossa - PR - Brasil

v. 07 , n. 01 , jan./mar. 2015 , p. $20-26$

DOI: $10.3895 /$ rbqv.v7n1.1976

\section{REVISTA BRASILEIRA DE QUALIDADE DE VIDA}

\title{
Relação dos níveis de burnout entre professores e diversas profissões no Brasil: um estudo comparativo
}

\section{Compared levels of burnout among teachers and various professions in Brazil: a comparative study}

\author{
Yonel Ricardo de Souza \\ Universidade Federal de Rondônia - UNIR - Porto Velho - Rondônia - Brasil \\ yonel@bol.com.br \\ Luis Alberto Lourenço Matos \\ Universidade Federal de Rondônia - UNIR - Porto Velho - Rondônia - Brasil \\ lumatospvh@hotmail.com
}

\section{RESUMO}

OBJETIVO: Correlacionar os níveis de burnout entre a profissão de professor e várias profissões que apresentam estudos do fenômeno no Brasil.

MÉTODOS: Foi realizado levantamento em periódicos alocados nas bases indexadoras LILACS e SCIELO e em dissertações e teses produzidas em programas de pós-graduação brasileiros. Dentre os itens listados, selecionaram-se aqueles trabalhos experimentais, quantitativos, que tivessem pesquisado o fenômeno burnout durante o exercício de uma profissão no Brasil. Para a análise de dados foi utilizado o pacote estatístico SPSS versão 20.0. Para verificar a relação entre as dimensões da síndrome de burnout, foi utilizada a correlação de Spearman's rho.

RESULTADOS: Observaram-se apenas correlações significativas $(\mathrm{p}<0,05)$ dos professores com atendente de vítima de violência (correlação inversa) e policial militar (correlação direta). As profissões funcionário público, motorista, funcionário de instituto de pesquisa, médico, enfermeiro e atleta de vôlei, além de um estudo que se valeu de uma amostra multifatorial (várias profissões), apresentaram níveis moderados de correlação com a profissão professor, entretanto sem significância.

CONCLUSÕES: Os fatores desencadeantes de estresse crônico entre as profissões com boa correlação com a profissão professor (principalmente policial militar) podem ser levados em consideração na montagem de estratégias para evitar a manifestação do burnout no trabalho, contribuindo assim para a promoção da saúde mental no trabalho e melhora da qualidade de vida.

PALAVRAS-CHAVE: Burnout. Profissão. Professor. Trabalho. Correlação.

\section{ABSTRACT}

OBJECTIVE: Correlate the levels of burnout among the teaching profession and various professions that present phenomenon of studies in Brazil. 
METHODS: Survey was carried out in allocated indexing journals in LILACS and SCIELO and dissertations and theses produced in Brazilian graduate programs. Among the items listed were selected ones, quantitative, experimental studies that have researched the phenomenon burnout during exercise of a profession in Brazil. For the data analysis the statistical package SPSS version 20.0 was used. To verify the relationship between the dimensions of burnout, the correlation Spearman'rho was used.

RESULTS: Were observed only significant correlations $(\mathrm{p}<0.05)$ of teachers with attendant victim of violence (inverse correlation) and military police (direct correlation). The civil servant, driver, official research institute, medical, nurse and volleyball player professions, plus a study that made use of a multifactorial sample (various professions), showed moderate levels of correlation with the teacher profession, however no significance.

CONCLUSIONS: The triggering factors of chronic stress among professions with good correlation with the teacher (mainly military police) profession can be taken into account in setting strategies to prevent the manifestation of burnout at work, thus contributing to the promotion of mental health at work and improved quality of life.

KEYWORDS: Burnout. Professional. Teacher. Job. Correlation.

\section{Introdução}

A anamnese do sofrimento e/ou adoecimento provocado ou desencadeado pelo trabalho e suas consequências, como o estresse, tem sido alvo de discussão e pesquisas entre vários profissionais da saúde (RODRIGUES; ALVARO; RONDINA, 2006; CARLOTTO; CÂMARA, 2007; DEJOURS, 2009). Antoniazzi, Dell'Aglio e Bandeira (1998) citam que os esforços despendidos por indivíduos para lidar com situações estressantes, crônicas ou agudas têm se constituído objeto de estudo da psicologia social, clínica e da personalidade, encontrando-se fortemente atrelados aos estudos das diferenças individuais.

Benevides-Pereira (2002), em um dos seus estudos, aponta que aspectos individuais ligados às condições de trabalho podem causar consequências emocionais e/ou físicas no relacionamento interpessoal e na capacidade de trabalho, podendo, em seu estágio final, levar o trabalhador a abandonar o emprego. Segundo Dejours (2009), o trabalho pode ser uma fonte de equilíbrio para o sujeito quando permite a descarga de tensão. Do contrário, pode provocar sofrimento e descompensações psicológicas ou somáticas que poderão levar o indivíduo a uma doença psicossomática. Diante disso, a síndrome de burnout (ou simplesmente burnout) tem sido amplamente estudada nos campos psicológico e psiquiátrico em todo mundo.

O burnout é entendido como uma síndrome psicológica decorrente da tensão emocional crônica no trabalho. Trata-se de uma experiência subjetiva interna que gera sentimentos e atitudes negativas no relacionamento do indivíduo com o seu trabalho (insatisfação, desgaste, perda do comprometimento), minando o seu desempenho profissional e trazendo consequências indesejáveis para a organização como absenteísmo, abandono do emprego e baixa produtividade (BENEVIDESPEREIRA, 2002).

O burnout é caracterizado pelas dimensões exaustão emocional (EE), despersonalização (D) e diminuição da realização pessoal (RP) (TAMAYO, 1997). O mesmo autor caracteriza a exaustão emocional pelos sintomas de fadiga, de esgotamento emocional e de negativismo. A despersonalização é caracterizada pela frieza e pela dureza no trato com clientes e com colegas de trabalho. Já a diminuição da realização profissional apresenta-se sob a forma de sentimentos de incompetência e/ou percepção de desempenho insatisfatório.

Vários instrumentos específicos têm sido desenvolvidos para a mensuração do burnout em cada profissão, como o Questionário de Burnout para Professores-R (CBP-R) e o Inventário de Burnout de Oldenburg. Entretanto, o Maslach Burnout Inventory (MBI) é o instrumento mais 
utilizado atualmente no mundo. O MBI tem três versões aplicáveis a categorias profissionais: Human Services Survey (MBI-HSS), para as áreas de saúde/cuidadores ou serviços humano-sociais; Educator's Survey (MBI-ES), para educadores; e, General Survey (MBI-GS), para profissionais que não estejam em contato direto com o público-alvo, e duas validações no Brasil: a de Tamayo (1997) e a de Benevides-Pereira (2002). É autoaplicável e avalia as três dimensões do burnout (Exaustão Emocional, Despersonalização e Realização Profissional).

Vários estudos têm procurado investigar os níveis de estresse e burnout no trabalho docente (CARLOTTO; CÂMARA, 2008; GARCIA; BENEVIDES-PEREIRA， 2003; MAZON; CARLOTTO; CÂMARA, 2008; MOREIRA et al., 2009; ZAFFARI et al., 2009), nas suas mais variadas manifestações: ensino público e particular, universitário, entre gêneros, etc. Todavia, não foram encontrados, na literatura atual, estudos que correlacionassem os níveis de burnout entre os professores e as demais profissões no país. Cabe ressaltar que no estudo de Carlotto e Câmara (2007), com uma amostra multifatorial, a apresentação dos escores não está diferenciada entre as profissões investigadas.

Nesse cenário, o objetivo do presente estudo foi correlacionar os níveis de burnout entre a profissão de professor e várias profissões que apresentam estudos do fenômeno no Brasil.

\section{Método}

Foi realizado levantamento em periódicos alocados nas bases indexadoras LILACS e SCIELO e em dissertações e teses produzidas em programas de pós-graduação brasileiros. Os descritores utilizados foram: burnout, exaustão emocional e estresse crônico. Dentre os itens listados, selecionaram-se aqueles trabalhos experimentais, de cunho quantitativo, que tivessem pesquisado o fenômeno burnout durante o exercício de uma profissão no Brasil. Em seguida os trabalhos foram classificados de acordo com a área profissional pesquisada e o instrumento(s) utilizado(s).

Com fins de fornecer coerência ao estudo, apenas as pesquisas que se valeram do mesmo instrumento selecionado (MBI) foram correlacionadas entre si. Nas pesquisas que apresentaram valores absolutos dentro das dimensões, foi dividido o valor absoluto de cada dimensão pelo número de itens $(\mathrm{EE}=9, \mathrm{D}=5$ e $\mathrm{RP}=8)$ para obter-se a média. Foi usado como referência entre a classe de professores o trabalho de Garcia e Benevides-Pereira (2003), pois foi o único dos três selecionados que apresentou médias do MBI. Dentre os trabalhos de Kirchhof (2013), Pereira (2013) e Jodas e Haddad (2009), todos tratando do burnout na enfermagem, apenas as médias deste último foram consideradas, devido a sua amostra ser mais abrangente.

Para a análise de dados foi utilizado o pacote estatístico SPSS versão 20.0. Análises descritivas e inferências foram conduzidas considerando um nível de significância de $p<0,01$. Para verificar a relação entre as dimensões da síndrome de burnout foi utilizada a correlação de Spearman's rho.

\section{Resultados e discussão}

Seguindo os procedimentos prescritos, foram selecionados: uma tese, duas dissertações e 16 artigos científicos. A Tabela 1 apresenta o resultado da coleta de dados indexados separados por autor, profissão e índices de burnout divididos nas três dimensões do instrumento utilizado nas diversas profissões no Brasil: 
Tabela 1 - Pesquisas, profissões, autores e média das dimensões do MBI

\begin{tabular}{|c|c|c|c|c|c|}
\hline \multirow[t]{2}{*}{ Ordem } & \multirow[t]{2}{*}{ Autor(es) } & \multirow[t]{2}{*}{ Profissão } & \multicolumn{3}{|c|}{ Dimensões Burnout } \\
\hline & & & $\mathrm{EE}$ & $\mathrm{D}$ & $\mathrm{RP}$ \\
\hline 1 & Santos, Pereira e Carlloto (2010) & $\begin{array}{l}\text { Atendente de vítima de } \\
\text { violência }\end{array}$ & 1,63 & 1,63 & 4,02 \\
\hline 2 & Neves (2010) & Funcionários públicos & 2,67 & 1,13 & 4,05 \\
\hline 3 & $\begin{array}{c}\text { Trindade, Lautert, Beck, Amestoy } \\
\text { e Pires (2010) }\end{array}$ & Equipe saúde da família & \multicolumn{3}{|c|}{$\begin{array}{c}\text { Apresentou os dados da amostra com e } \\
\text { sem burnout }\end{array}$} \\
\hline 4 & Mazon, Carlotto e Câmara (2008) & Professores & \multirow{2}{*}{\multicolumn{3}{|c|}{$\begin{array}{c}\text { Não foram apresentados valores, apenas } \\
\text { correlações } \\
\text { Não foram apresentados valores, apenas } \\
\text { correlações }\end{array}$}} \\
\hline 5 & $\begin{array}{l}\text { Zaffari, Peres, Carlotto e Câmara } \\
\text { (2009) }\end{array}$ & Professores (entre gêneros) & & & \\
\hline 6 & Carlotto e Câmara (2008) & $\begin{array}{l}\text { Professores (públicos e } \\
\text { particulares) }\end{array}$ & & & \\
\hline 7 & $\begin{array}{c}\text { Moreira, Farias Both e Nascimento } \\
\text { (2009) }\end{array}$ & $\begin{array}{c}\text { Profissionais de educação } \\
\text { física }\end{array}$ & \multicolumn{3}{|c|}{$\begin{array}{l}\text { Apresentou apenas frequência e } \\
\text { percentual das dimensões }\end{array}$} \\
\hline 8 & Morais (2008) & Padres & & & \\
\hline 9 & Carlotto e Câmara (2007) & $\begin{array}{l}\text { Várias profissões (amostra } \\
\text { multifatorial) }\end{array}$ & 2,35 & 1,75 & 1,65 \\
\hline 10 & Lopes e Garcia (2012) & Motoristas & 2,61 & 2,20 & 3,50 \\
\hline 11 & Fernandes (2011) & $\begin{array}{l}\text { Funcionários de instituto de } \\
\text { pesquisa }\end{array}$ & 2,13 & 1,68 & 3,79 \\
\hline 12 & Kirchhof (2013) & Docentes enfermagem & 3,79 & 6,09 & 4,92 \\
\hline 13 & Bolzan (2012) & Residentes médicos & 2,59 & 2,25 & 3,35 \\
\hline 14 & $\begin{array}{c}\text { Campos, Trotta, Bonafé e Maroco } \\
\text { (2010) }\end{array}$ & Dentistas & \multicolumn{3}{|c|}{ Utilizou o OLBI* } \\
\hline 15 & Garcia e Benevides-Pereira (2003) & Professores universitários & 2,13 & 0,98 & 1,27 \\
\hline 16 & Rocha e Cavalcante Neto (2014) & Policiais militares & 2,12 & 1,22 & 1,95 \\
\hline 17 & Jodas e Haddad (2009) & Profissionais de enfermagem & 1,89 & 1,56 & 4,58 \\
\hline 18 & Pereira (2013) & Auxiliar de enfermagem & 2,70 & 1,81 & 3,81 \\
\hline 19 & $\begin{array}{l}\text { Vieira, Carruzo, Aizava e Rigoni } \\
\text { (2013) }\end{array}$ & Atletas de vôlei & 1,57 & 1,50 & 2,50 \\
\hline
\end{tabular}

Fonte: Autoria própria (2014).

Obs: *OLBI: Oldenburg Burnout Inventory.

Considerando as ocupações que seguiram os critérios estabelecidos no presente estudo, seguem na Tabela 2 os resultados encontrados. A matriz utilizada para a correlação teve como variáveis as profissões e como registros pareados as médias de cada dimensão do Burnout.

Tabela 2 - Correlação entre as profissões - MBI

\begin{tabular}{|c|c|c|c|c|c|c|c|c|c|c|}
\hline & & $\begin{array}{c}\text { Atendente } \\
\text { Vit viol }\end{array}$ & $\begin{array}{c}\text { Func } \\
\text { publico }\end{array}$ & $\begin{array}{l}\text { Multi } \\
\text { fatorial }\end{array}$ & Motorista & $\begin{array}{c}\text { Func } \\
\text { Inst } \\
\text { pesq }\end{array}$ & Médico & $\begin{array}{c}\text { Policial } \\
\text { militar }\end{array}$ & Enfermeir & $\begin{array}{c}\text { Atleta } \\
\text { volei }\end{array}$ \\
\hline \multirow[t]{2}{*}{ Professor } & $\begin{array}{l}\text { Coeficiente de } \\
\text { Correlação }\end{array}$ & 0,000 & 0,500 & 0,500 & 0,500 & 0,500 & 0,500 & 1,000 & 0,500 & 0,500 \\
\hline & Significância* & 1,000 & 0,667 & 0,667 & 0,667 & 0,667 & 0,667 & - & 0,667 & 0,667 \\
\hline
\end{tabular}

De acordo com o modelo teórico de Maslach (1982), o burnout é um processo onde a dimensão precursora da síndrome se inicia com a exaustão emocional, seguida por despersonalização e, finalizando pelo sentimento de diminuição da realização pessoal no trabalho. 
Desta maneira, os resultados coletados em todas as profissões selecionadas apontam para a manifestação de uma dinâmica psicossomática em curso (progressão).

Aplicando o teste de correlação de Spearman, observaram-se apenas correlações significativas $(\mathrm{p}<0,05)$ dos professores universitários com atendente de vítima de violência (correlação inversa) e policial militar (correlação direta). Da análise da publicação de Rocha e Cavalcante Neto (2014) e da correlação direta encontrada no presente estudo, há indícios que as condições de trabalho dos professores se assemelhem aos policiais militares, como carga horária de trabalho elevada, baixos salários, rotina de atividade física no trabalho abaixo do recomendado e imposições previstas em regulamento interno, que podem levar o profissional a ter problemas de saúde mental.

De forma inversa, a ocupação atendente de vítima de violência obteve a menor correlação entre as estudadas (correlação inversa). As profissões funcionário público, motorista, funcionário de instituto de pesquisa, médico, enfermeiro e atleta de vôlei apresentaram correlação moderada, mais sem significância estatística, pois os valores de $\mathrm{p}$ apresentados na coluna significância são maiores que 0,05. Desta forma, não é possível afirmar com segurança que os fatores desencadeantes da exaustão emocional, despersonalização e baixa realização profissional na área da docência podem ser considerados nas demais profissões.

\section{Considerações finais}

Os achados do presente estudo permitem visualizar, ainda que de forma limitada, a síndrome de burnout no interior da profissão professor e compará-la com outros estudos que pesquisaram o mesmo fenômeno utilizando o MBI no Brasil. Fatores estressores correlacionáveis das profissões como carga horária de trabalho elevada, baixos salários, insuficiente atividade física e imposições previstas em regulamento interno podem ser fruto de agentes estressores similares (ambiente, liberdade, plano de carreira, entre outros).

Os fatores desencadeantes de estresse crônico nas profissões com boa correlação com a profissão professor podem ser levados em consideração na montagem de estratégias para evitar a manifestação do burnout no trabalho, contribuindo assim para a diminuição dos seus efeitos.

As limitações do trabalho se devem ao fato de não haver uma unidade do instrumento utilizado nos diversos trabalhos e alguns, embora citem o instrumento, não apresentam as médias dos índices nos resultados. Mais estudos devem ser realizados, entretanto, para a difusão do leque de conhecimentos sobre o burnout no trabalho no Brasil, valendo-se, se possível, de um instrumento unificado. Entendendo-se a dinâmica psicossomática do burnout, podem-se desenvolver estratégias de enfrentamento (coping) de forma eficiente, diminuindo os níveis de estresse no trabalho e contribuindo para a melhoria da qualidade de vida no trabalho.

\section{Referências}

ANTONIAZZI, A. S.; DELL'AGLIO, D. D.; BANDEIRA, D. R. O conceito de coping: uma revisão teórica. Estudos de Psicologia, v. 3, n. 2, p. 273-294, 1998. rossef

BENEVIDES-PEREIRA, A. M. T. Burnout: quando o trabalho ameaça o bem-estar do trabalhador. São Paulo: Casa do Psicólogo. 2002.

BOLZAN, M. E. O. Estresse, coping, síndrome de burnout, sintomas depressivos e hardiness em residentes médicos. 132 f. 2012. Dissertação (Mestrado em Enfermagem) - Programa de Pósgraduação em Enfermagem, Universidade Federal de Santa Maria, Santa Maria, 2012. 
CAMPOS, J. A. D. B.; TROTTA, O. S. H.; BONAFÉ, F. S. S.; MAROCO, J. Burnout em dentistas do serviço público: ter ou não ter, eis a questão! Revista de Odontologia da UNIFESP, v. 39, n. 10, p. 109-114, 2010.

CARLOTTO, M. S.; CÂMARA, S. G. Propriedades psicométricas: o Maslach Burnout Inventory em uma amostra multifatorial. Estudos de Psicologia, v. 24, n. 3, p. 325-332, 2007. rossef

CARLOTTO, M. S.; CÂMARA, S. G. Síndrome de burnout e estratégias de enfrentamento em professores de escolas públicas e privadas. Psicologia da Educação, v. 26, p. 29-46, 2008.

DEJOURS, C. Psicodinâmica do trabalho: contribuições da escola dejouriana à análise da relação prazer, sofrimento e trabalho. São Paulo: Atlas, 2009.

FERNANDES, G. Clima organizacional, Síndrome de Burnout, estratégias de enfrentamento no trabalho em funcionários de instituto de pesquisa do Vale do Paraíba paulista. 174 f. 2011. Dissertação (Mestrado em Gestão e Desenvolvimento Regional) - Programa de Pós-Graduação em Administração, Universidade de Taubaté, Taubaté, 2011.

GARCIA, L. P.; BENEVIDES-PEREIRA, A. M. T. Investigando o burnout em professores universitários. Revista Eletrônica InterAçãoPsy, v. 1, p. 76-89, 2003.

JODAS, D. A.; HADDAD, M. C. A. Síndrome de burnout em trabalhadores de enfermagem de um pronto socorro de hospital universitário. Acta Paulista de Enfermagem, v. 22, n. 2, p. 192-197, 2009. crossef

KIRCHHOF, R. S. Estresse, coping, síndrome de burnout, sintomas depressivos e hardiness em docentes de enfermagem. 150 f. 2013. Dissertação (Mestrado em Enfermagem) - Programa de Pós-Graduação em Enfermagem, Universidade Federal de Santa Maria, Santa Maria, 2013.

LOPES, M. O.; GARCIA, F. O. Síndrome de burnout: um estudo com pequenas empresas de transporte de turismo rodoviário na região de Contagem-MG. FACEF - Pesquisa,

Desenvolvimento e Gestão, v. 15, n. 1, p. 76-89, 2012.

MASLACH, C. Burnout: the cost of caring. New Jersey: Prentice Hall, 1982.

MAZON, V.; CARLOTTO. M. S.; CÂMARA, S.. Síndrome de burnout e estratégias de enfrentamento em professores. Arquivos Brasileiros de Psicologia, v. 60, n. 1, p. 55-66, 2008.

MORAIS, M. F. A. Stress, coping e burnout em padres responsáveis pela formação de seminaristas católicos. 182 f. 2008. Tese (Doutorado em Ciências da Religião) - Programa de Pósgraduação em Ciências da Religião, Pontifícia Universidade Católica, São Paulo, 2008.

MOREIRA, H. R.; FARIAS, G. O.; BOTH, J.; NASCIMENTO, J. V. Qualidade de vida no trabalho e síndrome de burnout em professores de educação física do estado do Rio Grande do Sul, Brasil. Revista Brasileira de Atividade Física \& Saúde, v. 14, n. 2, p. 115-122, 2009.

NEVES, M. O. M. Síndrome de burnout e estratégias de enfrentamento do estresse no trabalho em funcionários em uma empresa pública. 133 f. 2010. Monografia (Graduação em Psicologia) Centro Universitário de Brasília, Brasília, 2010. 
PEREIRA, S. S. Incidência de síndrome de burnout em técnicos e auxiliares de enfermagem e sua associação com o estresse precoce e estratégias de enfrentamento. 130 f. 2013. Dissertação (Mestrado em Ciências) - Programa de Pós-graduação em Enfermagem Psiquiátrica, Universidade de São Paulo, Ribeirão Preto, 2013.

ROCHA, D. F.; CAVALCANTE NETO, J. L. A síndrome de burnout e os níveis de atividade física em policiais militares ambientais de Alagoas. Revista Brasileira de Qualidade de Vida, v. 6, n. 1, p. 27-37, 2014. Crossef

RODRIGUES, P. F.; ALVARO, A. L. T.; RONDINA, R. Sofrimento no trabalho na versão de Dejours. Revista Científica Eletrônica de Psicologia, v. 7, p. 1-8, 2006.

SANTOS, C.; PEREIRA, K. W.; CARLOTTO, M. G. Burnout em profissionais que trabalham no atendimento de vítimas de violência. Barbaroi, v. 32, p. 69-81, 2010.

TAMAYO, R. M. Relação entre a síndrome de burnout e os valores organizacionais no pessoal de enfermagem de dois hospitais públicos. 126 f. 1997. Dissertação (Mestrado em Psicologia) Programa de Pós-graduação em Psicologia, Universidade de Brasília, Brasília, 1997.

TRINDADE, L. L.; LAUTERT, L.; BECK, C. L. C.; AMESTOY, S. C.; PIRES, D. E. P. Estresse e síndrome de burnout entre trabalhadores da equipe de Saúde da Família. Acta Paulista de Enfermagem, v. 23, n. 5, p. 684-689, 2010. Crossef

VIEIRA, L. F.; CARRUZO, N. M.; AIZAVA, P. V. S.; RIGONI, P. A. G. Análise da síndrome de burnout e das estratégias de coping em atletas brasileiros de vôlei de praia. Revista Brasileira de Educação Física e Esporte, v. 27, n. 2, p. 269-276, 2013. Croșef

ZAFFARI, N. T.; PERES, V. L.; CARLOTTO, M. S.; CÂMARA, S. C. Síndrome de burnout e estratégias de coping em professores: Diferença entre gêneros. Psicologia IESB, v. 1, n. 2, p. 1-12, 2009. 